\title{
Nonlinear Liquid Drop Model. Cnoidal Waves
}

\author{
Andrei Ludu ${ }^{1}$, Aureliu Săndulescu ${ }^{2}$ and Walter Greiner \\ Institut für Theoretische Physik der J.W.Goethe Universität, \\ D-60054 Frankfurt am Main, Germany
}

\begin{abstract}
By introducing in the hydrodynamic model, i.e. in the hydrodynamic equations and the corresponding boundary conditions, the higher order terms in the deviation of the shape, we obtain in the second order the Korteweg de Vries equation $(\mathrm{KdV})$. The same equation is obtained by introducing in the liquid drop model (LDM), i.e. in the kinetic, surface and Coulomb terms, the higher terms in the second order. The KdV equation has the cnoidal waves as steady-state solutions. These waves could describe the small anharmonic vibrations of spherical nuclei up to the solitary waves. The solitons could describe the preformation of clusters on the nuclear surface. We apply this nonlinear liquid drop model to the alpha formation in heavy nuclei. We find an additional minimum in the total energy of such systems, corresponding to the solitons as clusters on the nuclear surface. By introducing the shell effects we choose this minimum to be degenerated with the ground state. The spectroscopic factor is given by the ratio of the square amplitudes in the two minima.
\end{abstract}

PACS numbers: 23.60.+e, 21.60.Gx, 24.30.-v, 25.70.ef .

\footnotetext{
${ }^{1}$ Permanent Address: Bucharest University, Bucharest-Magurele, PO.Box MG-5211, Romania; email:ludu@th.physik.uni-frankfurt.de

${ }^{2}$ Permanent Address:Institute of Atomic Physics, Department of Theoretical Physics, BucharestMagurele, P.O.Box MG-6, Romania; e-mail:sandulescu@roifa.ifa.ro and Romanian Academy, Calea Victoriei 125, 71102 Bucharest, Romania; E-mail: aursand@aix.acad.ro
} 


\section{Introduction}

It is well known that liquid drop model, as a collective model of the nucleus describes excelently the spectra of spherical nuclei as small vibrations (harmonic in the linear approximation or anharmonic in higher approximations) around their shape. On the other hand it is known that on the nuclear surface of heavy nuclei close to the magic nuclei $\left({ }^{208} \mathrm{~Pb},{ }^{100} \mathrm{Sn}\right)$ a large enhancement of clusters (alpha, carbon, oxigen, neon, magnesium, silicon) exists which leads to the emission of such clusters as natural decays $[1,2]$. It is also clear that traditional collective models [3] are not able to give a complete explanation of such natural decays, i.e. they still did not completely answer to the main physical question: why should nucleons join together and spontaneous form an isolated cluster on the nuclear surface ? Only by the introduction in the shell model of many body correlation effects we could form an isolated bump, stable in time. It is possible to describe the formation of such clusters in a collective model $[4,5]$ ?

In the present paper by introducing the nonlinearities in the liquid drop model we succeded to give a positive answer to this problem. In a nonlinear liquid drop model we can describe simultaneously, by cnoidal waves the transitions from small vibrations to the formation of solitons. The experimental discovery and the theoretical foundation of solitons [6] as non-dispersive localized waves moving uniformly, lead to a powerfull theory of classical field equations with such solutions [7-9]. Also it was possible to explain the correspondence between classical soliton solutions and the extended-particle states of the quantized version of the theory. This lead to a generalisation of the semiclassical expansion of quantum mechanics in quantum field theory. In the last case solitons (and breathers or instanton solutions) are non-perturbative. Of course, the above methods work only if the initial physical model allows first the existence of some non-trivial classical localized solutions.

From the mathematical physics point of view solitons, as solutions of non-linear evolution equations, are isolated waves which preserve their shape and have finite and localized energy density. Recently has been shown that solitons may be also relevant 
in nuclear physics [4,5] or particle physics [10]. Also it was realized that many field theoretical models for particle interactions, and even for quantum extended particles [11], possess soliton or breather solutions and that the solitons ought to be interpreted as additional particle-like structures in theory. The traveling solutions of the KdV equation [7] and singular solutions having poles at $\pm \infty$. Recently, in order to describe the quasimolecular spectra, we have introduced in [5] a one-dimensional soliton model for the cluster (alpha particle) and the rotator-vibrator model for the nucleus. An excelent agreement with the experimental data was obtained.

We conclude that the nonlinear terms lead to new qualitative picture of the liquid drop model, i.e. the harmonic oscillations grow into anharmonic ones which can lead to a stable soliton configuration. Both the potential picture and the phase space portraits support this behaviour. We stress that all these results are embedded into a Hamiltonian formalism.

In the present paper we first introduce in the hydrodynamical model the higher terms in the deviation of the spherical shape. We have shown that in the third order we obtain the Korteweg de Vries equation $(\mathrm{KdV})$. In the following chapter 3 by introducing in the liquid drop model the third order terms in the deviation of the shape we obtained the same $\mathrm{KdV}$ equation as in the nonlinear hydrodynamical model. We should like to stress that these nonlinear equations are Hamiltonian eqautions which describe the total energy of the system. Chapter 4 contains the nonlinear solutions: cnoidal waves and the singular solutions for $\mathrm{KdV}$ equation. Last chapter discuss the application of nonlinear liquid drop model to the alpha preformation factors.

\section{The nonlinear hydrodynamic model}

There are two possible ways to describe the classical dynamics of a liquid drop: first is the fluid (hydrodynamic) approach based on the continuity, Euler and the equations of state together with boundary conditions and the second is the Hamiltonian approach. However there is a deep connection between these two approaches. The motion of a 
perfect incompressible liquid in a domain is governed only by the Euler equation, since the continuity and state equations reduce in this case to the Poisson equation. The boundary conditions ask the inner product between the velocity field and the volume differentiable 3 -form to be zero (special procedure occures when the boundary itself is variable in time - free surface - and results in nonlinear contributions to the differential equations). The flow of the velocity field belongs to the group of volume preserving transformations of the volume of the drop and hence it could be a geodesic on the manifold of this group. Now, the connection with the second approach comes from the observation that the Euler equation is Hamiltonian in the sense that, between all other possible flows, only those which satisfy Euler equation are geodesics. In the case of the liquid drop model the dominant terms are the volume, the surface and Coulombian. All terms are dependent on the geometry of the surface. Therefore, we expect that the dynamics of the liquid drop is strongly dominated (phenomenologicaly) by the dynamics of the free surface, in both these approaches.

Let us describe the surface of the nucleus in spherical coordinates $(r, \theta, \phi)$ as a function of the polar angles $\theta$ and $\phi$, by writing the nuclear radius in the form

$$
r=R_{0}(1+\xi(\theta, \phi, t)),
$$

where $R_{0}$ is the radius of the spherical nucleus and the shape function $\xi$ is the difference in the radius between the deformed and the spherical one. In the following we search for deformed surfaces which contain stable traveling waves which can lead to a bump. The stability is fulfilled if the function describing the surface arises as a solitary wave solution of one of the classical nonlinear equations like KdV (Korteweg de Vries), whose stability was clearly established in literature $[7,12,13]$. These equations are tractable as dynamical systems in the frame of nonlinear infinite-dimensional Hamiltonian field theories. The cnoidal waves as solutions of KdV equation describe small vibrations up to the solitary waves. In the case of the spherical surface, the localization condition is realised for values of the angular half-width $L$ of the shape function $\xi(\theta, \phi, t)$ smaller than $\pi$, at any moment of time $[4,5]$. Without any loss of generality and in the spirit of the above theory, it is convenient to look for a special space-time behaviour of the 
shape function of the form

$$
\xi(\theta, \phi, t)=g(\theta) \eta(\phi-V t)
$$

with $g$ an arbitrary bounded, non-vanishing continuous function, $\eta$ a compact supported, or rapidly decreasing function with $V$ defining the tangential velocity of the traveling solution $\eta$ on the surface. This solution represents a stable traveling perturbation $(\eta)$ in the $\phi$ direction, having a given transversal profile $(g)$ in the $\theta$ direction. This is different from the traditional liquid drop model case when one expands the shape function in spherical harmonics and where we have shown [5] one needs to consider more than 10 multipoles to fit such shapes of a localised bump.

We suppose (without any loose of generality, due to the spherical symmetry) that the bumps are situated on a circle $\theta=\pi / 2$ such that the variable $\theta$ plays only the role of a parameter in the corresponding dynamical equations, and that the bumps travel along the $\phi$ coordinate only. This choice results in the separation of variables in the shape function in eq.(2). Since in the investigation of the dynamics of the surface the coordinate $r$ will be involved only in the (free surface) boundary conditions, then $\phi$ remains the unique free coordinate. Hence, we can reduce the whole 3-dimensional problem to a 1-dimensional formalism.

In the hydrodynamic approach we treat the nucleus is a perfect ideal fluid layer (incompressible, irrotational and without viscosity) described by the field velocity $\vec{v}(r, \theta, \phi, t)$ and by the constant mass density $\rho=$ const. From the continuity equation we have div $\vec{v}=0$ and due to the irrotationality condition, $\nabla \times \vec{v}=0$, we have a potential flow, described by the velocity potential $\Phi(r, \theta, \phi, t)$, and the corresponding Laplace equation

$$
\vec{v}=\nabla \Phi, \quad \triangle \Phi=0
$$

The dynamics of this perfect fluid is described by the Euler equation

$$
\frac{\partial \vec{v}}{\partial t}+(\vec{v} \cdot \nabla) \vec{v}=-\frac{1}{\rho} \nabla P+\frac{1}{\rho} \vec{f}
$$

where $P$ is the pressure and $\vec{f}$ is the volume density of the forces acting into this fluid, e.g. for the Coulombian one we have $\vec{f}=-\rho_{e l} \nabla \Psi$, with $\Psi$ the electrostatic potential 
and $\rho_{e l}$ the charge density, supposed to be constant, too. By using eqs.(3), eq.(4) becomes, in the Coulombian case

$$
\left.\left(\Phi_{t}+\frac{1}{2}|\nabla \Phi|^{2}\right)\right|_{\Sigma}=-\frac{1}{\rho} P-\left.\frac{\rho_{e l}}{\rho} \Psi\right|_{\Sigma}
$$

To determine uniquely the unknown functions $\Phi$ and $\xi$ we need, in completion of eqs. $(3,5)$, the boundary conditions for the scalar harmonic field $\Phi$, on (maximum) two closed surfaces: the external free surface of the nucleus described by eqs.(1) or (2) and the inner surface (if it exists) of the fluid layer. The latter condition can be expressed in a simpler form if we consider that the motion is limited to only a thin fluid layer characterised by zero radial velocity of the flow on its inner surface. This last condition expresses the existence of a rigid core in the volume of the nucleus. The first boundary condition can be expressed in the most general form of the kinematical constrain of the free surface of the fluid described by eq.(1), $[4,5,7]$

$$
\left.\frac{d r}{d t}\right|_{\Sigma}=\left.\left(\frac{\partial r}{\partial t}+\frac{\partial r}{\partial \theta} \frac{d \theta}{d t}+\frac{\partial r}{\partial \phi} \frac{d \phi}{d t}\right)\right|_{\Sigma}
$$

where $r(t, \phi, \theta)$ in the RHS represents the shape function described in eq.(1) and the label $\Sigma$ means that eq.(6) is taken on the free surface $\Sigma$. This equation allows very general types of movements, including traveling and vibrational waves. Eq.(6) reduces to the form $\left.\frac{d r}{d t}\right|_{\Sigma}=\left.\frac{\partial r}{\partial t}\right|_{\Sigma}$ when one considers only its linear approximation [3,14], i.e. that one used in the Bohr-Mottelson model. This linearization implies the existence of only collective radial vibrations and does no allow any motion along the tangential direction. Eq.(6) can be written in terms of the derivatives of the potential of the flow and the shape function $\xi$

$$
\left.\Phi_{r}\right|_{\Sigma}=\left.R_{0}\left(\xi_{t}+\frac{\xi_{\theta}}{r^{2}} \Phi_{\theta}+\frac{\xi_{\phi}}{r^{2} \sin ^{2} \theta} \Phi_{\phi}\right)\right|_{\Sigma}
$$

where $\frac{\partial \Phi}{\partial r}=v_{r}=\dot{r}$ is the radial velocity and $\frac{1}{r} \frac{\partial \Phi}{\partial \theta}=v_{\theta}=r \dot{\theta}, \frac{1}{r \sin \theta} \frac{\partial \Phi}{\partial \phi}=v_{\phi}=r \dot{\phi} \sin \theta$ are the tangential velocities. We denote here the partial differentiation by suffixes, $\partial \Phi / \partial \phi=\Phi_{\phi}$, etc. The existence of a rigid core of radius $R_{0}-h(\theta)>0, h(\theta) \ll R_{0}$, introduces the second boundary condition for the radial velocity on the surface of this core in the form

$$
\left.v_{r}\right|_{r=R_{0}-h}=\left.\frac{\partial \Phi}{\partial r}\right|_{r=R_{0}-h}=0 .
$$


Both eqs. $(7,8)$ are von Neumann type of boundary conditions. The motion of the fluid is described by the Laplace equation eq.(3) for $\Phi$, and by the two boundary conditions, eqs. $(7,8)$, for $\Phi$ and $\xi$. To these equations we have to add the dynamical equation in the form of Euler-Lagrange equation if we use a Lagrangean formalism, or in the form of Hamilton equation if we use a Hamiltonian formalism. In the present paper we consider the contribution of the nonlinear terms in all equations (e.g. the second and third terms of RHS of eqs. $(6,7))$. The corresponding solutions should reduce to the standard normal modes of vibrations, if we restrict to the linear approximation.

For such typical hydrodynamical problems, like that described by eqs. $(3,5,7,8)$, one generaly uses, in the linear approximation, the expansion in spherical harmonics. Such an expansion is no more appropriate for the nonlinear cases. Hence, we use for the potential of the flow the expansion

$$
\Phi=\sum_{n=0}^{\infty}\left(\frac{r-R_{0}}{R_{0}}\right)^{n} f_{n}(\theta, \phi, t)
$$

where the functions $f_{n}$ are not orthogonal on the surface of the sphere and do not form in general a complete system. The convergence of eq.(9) is controlled by the value of the small quantity $\frac{r-R_{0}}{R_{0}} \leq \max |\xi|=\epsilon$, [4]. From the Laplace equation (in spherical coordinates), and the expansions

$$
\frac{1}{r^{n}}=\frac{1}{R_{0}^{n}} \sum_{k=0}^{\infty}(-1)^{k}((n-1) k+1) \xi^{k}, \quad k=1,2,
$$

we obtain a system of equations which result in the recurrence relations for the unknown functions $f_{n}$

$f_{n}=\frac{(-1)^{n-1}(n-1) \triangle_{\Omega} f_{0}-2(n-1) f_{n-1}+\sum_{k=1}^{n-2}(-1)^{n-k}\left(2 k-(n-k-1) \triangle_{\Omega} f_{k}\right)}{n(n-1)}$

with $n \geq 2$ and where $\triangle_{\Omega}=\frac{1}{\sin \theta} \frac{\partial}{\partial \theta}\left(\sin \theta \frac{\partial}{\partial \theta}\right)+\frac{1}{\sin ^{2} \theta} \frac{\partial}{\partial \phi}$ is the angular part of the Laplacean operator in spherical coordinates. Eq. (11) reduces the unknown functions to only two: $\triangle_{\Omega} f_{0}$ and $f_{1}$ :

$$
\begin{gathered}
f_{2}=-\frac{1}{2}\left(\triangle_{\Omega} f_{0}+2 f_{1}\right), \\
f_{3}=\frac{1}{6}\left(4 \triangle_{\Omega} f_{0}-4 \triangle_{\Omega} f_{1}+4 f_{1}+2\right),
\end{gathered}
$$




$$
f_{4}=\frac{1}{24}\left(\triangle_{\Omega}^{2} f_{0}-14 \triangle_{\Omega} f_{0}+8 \triangle_{\Omega} f_{1}-8 f_{1}\right) \quad \ldots
$$

If we choose the independent functions $\triangle_{\Omega} f_{0}$ and $f_{1}$ to be smooth on the sphere, they must be bounded together with all the $f_{n}$ 's (these being linear combinations of higher derivatives of $f_{0}$ and $f_{1}$ ) and hence the series in eq. (9) are indeed controlled by the difference in the radius between the deformed and the spherical one. However, in the following we will use only truncated polynomials of these series.

By introducing eqs.(11-12) in the second boundary condition, eq.(8), we obtain the condition

$$
\sum_{n=1}^{\infty} n\left(-\frac{h}{R_{0}}\right)^{n-1} f_{n}=0
$$

which reads, in the first order in $h / R_{0}$

$$
f_{1}=\frac{2 h}{R_{0}} f_{2}
$$

From eqs. $(12,14)$ the unknown function $f_{1}$ is obtained, in the smallest order in $h / R_{0}$

$$
\triangle_{\Omega} f_{0}=-\left(\frac{R_{0}}{h}+2\right) f_{1} .
$$

Concerning the first boundary condition held at the free surface $\Sigma$, eq.(7), we need to calculate the derivatives of the potential of the flow on that surface

$$
\begin{gathered}
\left.\Phi_{r}\right|_{\Sigma}=\sum_{n} n \frac{\left(r-R_{0}\right)_{\Sigma}^{n-1}}{R_{0}^{n}} f_{n}=\frac{f_{1}}{R_{0}}+\frac{2 \xi f_{2}}{R_{0}}+\mathcal{O}_{2}(\xi), \\
\left.\Phi_{\phi}\right|_{\Sigma}=\sum_{n} \xi^{n} f_{n, \phi}=f_{0, \phi}+\xi f_{1, \phi}+\mathcal{O}_{2}(\xi), \\
\left.\Phi_{\theta}\right|_{\Sigma}=\sum_{n} \xi^{n} f_{n, \theta}=f_{0, \theta}+\xi f_{1, \theta}+\mathcal{O}_{2}(\xi) .
\end{gathered}
$$

By introducing the series eqs.(10,16) in eq.(7), for the traveling wave solution eq.(2), we have the equation

$$
f_{1}+2 \xi f_{2}=R_{0}^{2} \xi_{t}+\frac{\xi_{\phi}(1-2 \xi)}{\sin ^{2} \theta}\left(f_{0, \phi}+\xi f_{1, \phi}\right)+\xi_{\theta}(1-2 \xi)\left(f_{0, \theta}+\xi f_{1, \theta}\right) .
$$

We keep the nonlinearity of the boundary conditions, eqs. $(7,8,13)$, in the first order (i.e. the first order in the expression of $f_{0}$ and the second order in the expression of $f_{1}$ ). 
Consequently, in order to be consistent, it is enough to take the linear approximation of the solution for $f_{1}$ in eq.(17), like in the case of the normal modes of vibrations

$$
f_{1}=R_{0}^{2} \xi_{t}+\mathcal{O}_{2}(\xi)
$$

Hence, by introducing the linear approximation for $f_{1}$ (eq.(18)) in eq.(17)

$$
2 \xi f_{2}=\frac{1}{\sin ^{2} \theta}\left(-\xi_{\phi} f_{0, \phi}+\xi \xi_{\phi}\left(f_{1, \phi}-2 f_{0, \phi}\right)\right)+\xi \xi_{\theta}\left(f_{1, \theta}-2 f_{0, \theta}\right)
$$

and by taking the expression of $f_{2}$ from the recurrence relations, eq.(14) and $\triangle_{\Omega} f_{0}$ from eq.(15), we obtain the form of $f_{0}$, in the second order in $\xi$

$$
f_{0, \phi}=-\frac{R_{0}^{3} \sin ^{2} \theta}{h} \frac{\xi \xi_{t}}{\xi_{\phi}}(1+2 \xi)-\frac{\xi_{\theta} f_{0, \theta}}{\xi_{\phi}}+\mathcal{O}_{3}(\xi) .
$$

In the case of traveling wave profile of the form $\xi(\theta, \phi, t)=g(\theta) \eta(\phi-V t)$, which introduces the restriction $\xi_{\phi}=-V \xi_{t}$ and vanishes the tangential velocity in the $\theta$ direction, eq.(20) becomes

$$
f_{0, \phi}=\frac{V R_{0}^{3} \sin ^{2} \theta}{h} \xi(1+2 \xi)+\mathcal{O}_{3}(\xi)
$$

Eqs. $(18,20,21)$ describe, in the second order in $\xi$, the connection between the velocity potential (the flow) and the shape function, through the boundary conditions. This fact is a typical feature of nonlinear systems. The dependence of $\left.\Phi\right|_{\Sigma}$ on the polar angles, in the second order in $\xi$, has the form of a quadrupole in the $\theta$-direction and depends only on $\xi$ and its derivatives in the $\phi$-direction. For traveling wave profiles the tangential velocity in the direction of motion of the perturbation, $v_{\phi}=\Phi_{\phi} / r \sin \theta$ is proportional with $\xi$ in the first order

$$
v_{\phi}=\frac{2 V R_{0} \sin \theta}{h} \xi+\mathcal{O}_{2}(\xi)
$$

with the constant of proportionality being exactly the coupling parameter $\chi$ from our previous solitonic model, i.e. eq.(20) in [4]. This can be seen also from Figs. 1 and $2 \mathrm{a}$ in [4], where it is clearly stated the $1 / h$ dependence of $\chi$, for fixed velocity $V$, like in eqs.(21,22). Eqs.(20-22) are valid for any traveling wave shape functions, case which includes harmonic or anharmonic oscillations, solitons, breathers, cnoidal waves, etc. [7-9,13]. 
In order to obtain the dynamical equation for the surface $\Sigma$ we follow the usual formalism for the normal vibration of a sphere [14], corrected with the corresponding nonlinear terms $[4,5,7,8]$, i.e. we solve the Euler equation on the free surface with respect to the potential flow $\Phi$ and the shape function $\xi$, for given pressure, force fields and boundary conditions. The pressure at the free surface $\Sigma$ can be obtained from the surface energy of the deformed nucleus, $U_{S}$

$$
U_{S}=\sigma R_{0}^{2} \int_{0}^{2 \pi} \int_{0}^{\pi}(1+\xi) \sqrt{(1+\xi)^{2}+\xi_{\theta}^{2}+\frac{\xi_{\phi}^{2}}{\sin ^{2} \theta}} \sin \theta d \theta d \phi,
$$

where $\sigma$ is the pressure surface coefficient. Indeed, by expanding in series the square root in eq.(23) with respect to $\xi$, up to the third order, we obtain for the first variation of the functional $U_{S}$

$$
\delta U_{S}=\sigma R_{0}^{2} \int_{0}^{2 \pi} \int_{0}^{\pi}\left(2+2 \xi+\xi^{2}-\triangle_{\Omega} \xi+3 \xi^{2} \xi_{\theta} c t g \theta\right) \delta \xi \sin \theta d \theta d \phi+\mathcal{O}_{4}(\xi) .
$$

Following [14] the surface pressure on $\Sigma$ is given by the local curvature radius of the surface, and from the volume conservation we have

$$
\left.P\right|_{\Sigma}=\sigma\left(\frac{1}{R_{1}}+\frac{1}{R_{2}}\right)=\sigma R_{0}^{2} \frac{\delta a_{\xi}}{R_{0}^{3}(1+2 \xi)},
$$

where $R_{1,2}$ are the principal radii of curvature of the surface of the fluid and $\delta a_{\xi}$ is the term in the paranthesis in eq.(24). By introducing eq.(24) in eq.(25) we obtain the expression of the surface pressure as a function of the shape function

$$
\left.P\right|_{\Sigma}=\frac{\sigma}{R_{0}}\left(-2 \xi-4 \xi^{2}-\triangle_{\Omega} \xi+3 \xi \xi_{\theta}^{2} \operatorname{ctg} \theta\right)+\text { const } .
$$

The terms of order three in $\xi_{\phi, \theta}, \xi_{\phi, \phi}$ and $\xi_{\theta, \theta}$, could be neglected in eq.(25) due to the high localisation of the solution (the relative amplitude of the deformation $\epsilon$ is smaller than its angular halh-width $L, \xi \xi_{\phi \phi} / R_{0}^{2} \simeq \epsilon^{2} / L^{2} \ll 1$, etc.).

The Coulombian potential is given by a Poisson equation, $\triangle \Psi=\rho_{e l} / \epsilon_{0}$, with $\epsilon_{0}$ the vacuum dielectric constant. By using the same method like for $\Phi$, [4], we obtain in the second order for $\xi$, the form

$$
\left.\Psi\right|_{\Sigma}=\frac{\rho_{e l} R_{0}^{2}}{3 \epsilon_{0}}\left(1-\xi-\frac{\xi^{2}}{6}\right) .
$$


In order to write the Euler equation, eq.(5), for the above restrictions, we take the surface pressure from eq.(26), the velocity potential from eqs. $(9,14,18,21)$, and the Coulombian potential from eq.(27) and we obtain, in the second order in $\xi$ and the first order in its derivatives

$$
\begin{gathered}
\left.\Phi_{t}\right|_{\Sigma}+\frac{V^{2} R_{0}^{4} \sin ^{2} \theta}{2 h^{2}} \xi^{2}=\frac{\sigma}{\rho R_{0}}\left(2 \xi+4 \xi^{2}+\triangle_{\Omega} \xi-3 \xi^{2} \xi_{\theta} \operatorname{ctg} \theta\right) \\
+\frac{\rho_{e l}^{2} R_{0}^{2}}{3 \epsilon_{0} \rho}\left(\xi+\frac{\xi^{2}}{6}\right)+\text { const. }
\end{gathered}
$$

Neglecting all nonlinear terms in this expression, we obtain

$$
\left.\Phi_{t}\right|_{\Sigma}=-V f_{0, \phi}=-\frac{\sigma}{\rho R_{0}}\left(2 \xi+\triangle_{\Omega} \xi\right)-\frac{\rho_{e l}^{2} R_{0}^{2}}{3 \epsilon_{0} \rho} \xi+\text { const. }
$$

which, together with the linearised eq.(7), i.e. $\left.\Phi_{r}\right|_{\Sigma}=R_{0} \xi_{t}$, describes the normal modes of vibration of the liquid drop in the presence of the Coulombian field with spherical harmonics solutions for the angular part and complex exponential for the time dependence. This linear approximation represents exactly the reduction of the present model to the traditional liquid drop model.

In Eq.(28) is a nonlinear partial differential equation with respect to $\theta$ and $\phi$. By differentiating it with respect to $\phi$, by using eqs. $(15,18)$ and by re-ordering the terms, we obtain, in the second order

$$
A(\theta) \eta_{t}+B(\theta) \eta_{\phi}+C(\theta) g(\theta) \eta \eta_{\phi}+D(\theta) \eta_{\phi \phi \phi}=0,
$$

which is a Korteweg de Vries (KdV) equation with variable coefficients depending on $\theta$, as a parameter

$$
\begin{aligned}
& A=\frac{V R_{0}^{2}\left(R_{0}+2 h\right) \sin ^{2} \theta}{h} ; \quad B=-\frac{\sigma}{\rho R_{0}} \frac{(2 g+\triangle g)}{g}-\frac{\rho_{e l^{2}} R_{0}^{2}}{3 \epsilon_{0} \rho} ; \\
& C=8\left(\frac{V^{2} R_{0}^{4} \sin ^{4} \theta}{8 h^{2}}-\frac{\sigma}{\rho R_{0}}\right)-\frac{\rho_{e l}^{2} R_{0}^{2}}{9 \epsilon_{0} \rho} ; \quad D=-\frac{\sigma}{\rho R_{0} \sin ^{2} \theta},
\end{aligned}
$$

where by $\triangle g$ we understand only the action over $\theta$. First we want to make a qualitative analysis of the solutions of this nonlinear equation. The solutions can be clasificated 
by either using the algebraic hierachies and topological arguments $[7-9,12,13]$ or by using the phase space portrait of these solutions $[8,12]$. The latter way gives a simpler picture of the classes of admisible solutions and, in addition, characterize their periodic and bounded character. In order to comment on the traveling wave solutions eq.(2), we use the relation $\partial_{t}=-V \partial_{\phi}$, with $V$ a parameter to be determined. We can then integrate the resulting equation once and write it in the form: $\eta_{t t}=\left(V A-A_{0}\right) V^{2} / C \eta-$ $B V^{2} / 2 C \eta^{2}$. This is the Newton equation of motion for a one-dimensional particle described by the coordinate $\eta(t)$ in a "potential force" given by the RHS of the above expression. By integrating once this "force" we obtain the effective one-dimensional potential associated with this motion, $U(\eta, V)$, depending on $V$ as a parameter. For a given value of $V$ we can construct a certain phase space of this type of solutions. All possible solutions of the KdV equation are classified according with all admissible trajectories of this particle, under the action of the effective potential, in the phase space, [8,12], Figs.1. In Fig.1a we present the effective potential shape and the associate trajectories in the phase space for the $\mathrm{KdV}$ equation. The possible trajectories with constant energy $\left(E=U(\eta, V)+\frac{1}{2} \eta_{t}^{2}\right)$, are classified through the parameter energy, $E$. If we reduce to the linear approximation, the effective potential is quadratic and the solutions allow only harmonic oscillations, Fig1b. This situation represents the case of the traditional liquid drop model where only the harmonic oscillations are taken into account, and the governing equation becomes the Helmoltz equation, [3,15]. The KdV equation has a cubic potential energy, Fig1b. This is an example in which one can see directly the effect of the introduction of the nonlinearity: the harmonic oscillator effective potential gets a pocket and a saddle point, which is responsible for the new soliton solutions. Oscillations still exist and become anharmonic, being described by the cnoidal wave solutions of the $\mathrm{KdV}$ equation. At the superior boundary of the pocket, where the energy $E$ equals the potential energy at the saddle, the anharmonic oscillations become aperiodic and describe the soliton solution of the KdV equation. For values of the energy higher than the saddle (the soliton energy), or for smaller than the bottom of the valley, the solutions decay into instable, singular ones, having poles towards infinity. However, these residual solutions are not taken into account here since 
they are not traveling waves.

Eq.(30) has as solutions the cnoidal waves. The localised solution, solitons have the form

$$
\eta(\phi, t)=g(\theta) \operatorname{sech}^{2}\left(\frac{\phi-V(\theta) t}{L(\theta)}\right)
$$

where $g(\theta) \leq \eta_{0}=\epsilon R_{0}$ is the soliton amplitude, $V(\theta)$ is its angular velocity and $L(\theta)$ is its half-width. Due to the nonlinearity of the equation, these coefficients depend on the coefficients of the $\mathrm{KdV}$ equation, through the relations, $[7,8]$

$$
L(\theta)=\sqrt{\frac{12 D}{\eta_{0} C}} ; \quad V(\theta)=\frac{g(\theta) C+3 A}{3 B},
$$

in other words, a higher soliton moves faster and has a larger half-width. The soliton is extremely stable against perturbations in the sense of smooth modifications of the coefficients of the equations, initial conditions, introduction of small additional terms in the equation or when interacting with other solutions of this equation (scattering). Solitons have a constant traveling profile in time and an infinite number of integrals of motion $[7-9,12,13]$. Details concerning the explicit dependence of the soliton parameters, as functions of the coefficients of the $\mathrm{KdV}$ equation eq.(30), are given in extenso in [4].

In the following we analyse the stability of the steady-wave solutions of the $\mathrm{KdV}$ equation, eq.(30), against the dependence of its coefficients on $\theta$. In order to have a real traveling wave along the $\phi$-direction, the parameters of the soliton solution must fulfil the following restrictions:

1. The amplitude of the soliton and the depth of the layer must decrease from their maximum values, $\left(\eta_{0}, h\right)$ on the circle $\theta=\pi / 2$ towards 0 , when $\theta \rightarrow 0, \pi$. Then, the function $g(\theta)$ must increase, when $\theta: 0, \pi \rightarrow \pi / 2$, from 0 to $\eta_{0}$. The function $h(\theta)$ must increase when $\theta: 0, \pi \rightarrow \pi / 2$, from 0 to $h$. The numbers $\eta_{0}, h(\pi / 2)$ (i.e. the maximum amplitude of the soliton and the maximum depth of the layer) are free parameters, as have been shown in $[4,5]$.

2. The (angular) half-width $L$, eq.(33), must be constant:

$$
D(\theta)=\mathcal{C}_{1} C(\theta) g(\theta)
$$


3. The (angular) velocity $V$, eq.(33), must be also constant:

$$
D(\theta)=\mathcal{C}_{2} B(\theta)
$$

where $\mathcal{C}_{1,2}$ are constants. Taking into account the mutual relations between the parameter of the soliton solution and the coefficients of the KdV equation, eqs.(33) and the restrictions 1-3, we obtain a system of two differential equations, eqs. $(34,35)$, for the two unknown functions $L(\theta), V(\theta)$, with bilocal Cauchy conditions at the ends of $[0, \pi]$,

given by condition 1. Consequently we have for these functions a well defined boundary condition problem with unique solutions, depending parametricaly on $\eta_{0}, h(\pi / 2)$. Both differential equations, eqs. $(34,35)$, are well defined in $\theta[0, \pi]$ and have no poles.

\section{The nonlinear liquid drop model}

The liquid drop model has an infinite-dimensional Hamiltonian structure described by a nonlinear Hamiltonian function. Such systems can be treated in the same way as the finite-dimensional ones, excepting some difficulties related with the differentiability of the flow and with the definition of the symplectic structure, [12]. These occure, on one side, due to the fact that the vector fields are only densely defined (since we are dealing with partial differential equations) and, on the other side, due to the fact that the linear Hamiltonian and the nonlinear one could have different symplectic structures (different associated symplectic forms, phase spaces, etc.). If these difficulties are overcome, the theory of Hamilton equations and the invariants and conservation laws may be used, too.

The dynamics governing traveling waves and small (even anharmonic) oscillations in a perfect irrotational fluid, in $1+1$ space-time dimensions, can be described by a scalar field $\Phi(x, t)$ and a Hamiltonian $H_{[\Phi]}=\int_{D} h d x$ with the Hamiltonian density $h$

$$
H_{[\Phi]}=\int_{x_{1}}^{x_{2}}\left(\frac{1}{2} \Phi_{t}^{2}-\frac{1}{V^{2}}\left|\Phi_{x}\right|^{2}+F(\Phi)\right) d x
$$

where $x_{1,2}= \pm \infty$ for the flow along the line $(D=R)$, and $x_{1,2}=0, \pi(x \rightarrow \phi \in$ $D=[0, \pi])$, for a flow on a closed manifold. The first term in the integrand stays 
for the kinetic energy $(T)$ and the next two for the potential energy $(U)$, the last one describing the restoring forces in a general manner. Such potentials occur in quantum theory of self-interacting mesons where the function $F$ governs the nonlinear part of the interaction. The configuration space for this problem is some space of smooth real fields with the associated symplectic form generated by the usual $L^{2}$ inner product. This approach can also provide a Lagrangian $L=T-V=\int_{D} \mathcal{L} d x$ and the equation of motion is

$$
\Phi_{t}=\Phi_{t t}-V^{2} \Phi_{x x}-\partial_{x} \frac{d F}{d \Phi}
$$

This formalism provides periodical harmonic solutions in $x$ for $F=0$ and nonlinear waves for general $F$. In order to obtain localised solutions, we have to use another type of Hamiltonian, i.e. a $\mathrm{KdV}$ (or modified $\mathrm{KdV}$, etc.) one. This could be provided by the Hamiltonian

$$
H[\Phi]=\int_{D} h\left(\Phi, \Phi_{x}, \Phi_{x x}, \ldots\right) d x
$$

where $D$ is again the domain of the generic space coordinate $x,[9]$. The Hamiltonian vector field id given by $X_{H[\Phi]}=\frac{\partial}{\partial x} \frac{\delta h}{\delta \Phi}$, where $\delta / \delta \Phi$ is the functional derivative of $h$ with respect to the $\Phi$

$$
\frac{\delta h}{\delta \Phi}=\sum_{k \geq 0}(-1)^{k+j} \partial_{t}^{j} \partial_{\phi}^{k} \frac{\partial h}{\partial \Phi_{\phi}^{k} \Phi_{t}^{j}},
$$

with $\partial_{t}^{j}=\partial^{j} / \partial t^{j}, \Phi_{\phi}^{k}=\partial^{k} \Phi / \partial \phi^{k}$, etc. The corresponding Hamilton equation becomes

$$
\frac{\partial \Phi}{\partial t}=-\frac{\partial}{\partial x} \frac{\delta h}{\delta \Phi}
$$

i.e. $\Phi(x, t)$ are the integral curves of $X_{H_{[\Phi]}}$. Localised solutions must vanish at infinity for $D=R$, and must be periodical or rapidly decreasing functions for $D=[0, \pi]$. A symplectic structure on these spaces of functions is given by the skew-scalar product, [12] of two arbitrary functions $\Phi_{1}$ and $\Phi_{2}$

$$
\omega\left(\Phi_{1}, \Phi_{2}\right)=\frac{1}{2} \int_{D}\left(\int_{x_{2}}^{x}\left(\Phi_{1}(x) \Phi_{2}(y)-\Phi_{1}(y) \Phi_{2}(x)\right) d y\right) d x .
$$

However it is a qualitative difference between the two Hamiltonians, eqs.(36,38), occuring from their different symplectic geometries. This difference has consequences in 
the existence of two different phase spaces, and finaly, in the difficulty of smoothly connecting their characteristic solutions, i.e. linear oscillations and solitary waves.

The liquid drop model consists in the sum of the kinetic and potential energy of the fluid, $E=T+U$. All the terms in $E$ depend on two functions: the shape of the surface $\xi(\theta, \phi, t)$ and the potential flow $\Phi(r, \theta, \phi, t)$. In the following we use for the shape function the factorization given in eq.(2) and we consider only one canonical coordinate, $\phi$. In the following we consider only those solutions $\xi(\theta, \phi, t)$ in which the coordinate $\theta$ describing the transversal profile of the traveling wave is considered to be decoupled of $\phi$, eq.(2). All the terms dependent of $\theta$ will be absorbed in the coefficients of some integrals, and will become the parameters of the model. In this case the energy becomes a functional of $\eta$ only.

The potential energy $U$ has at least three terms: the surface energy $\left(U_{S}\right)$, the Coulombian energy $\left(E_{C}\right)$ and the shell energy $\left(E_{s h}\right)$. The first term describing the surface contribution is

$$
U_{S}=\sigma\left(\mathcal{A}_{\xi}-\mathcal{A}_{0}\right)
$$

where $\sigma$ is the surface pressure coefficient, $\mathcal{A}_{\xi}$ being the area of the deformed nucleus and $\mathcal{A}_{0}$ the area of the spherical nucleus, of radius $R_{0}$. Both these areas encircle the same volume $V_{0}=\frac{4 \pi R_{0}^{3}}{3}$ of the nucleus. The surface of the deformed nucleus depends only of the shape function $\xi(\theta, \phi, t)$, eqs. $(1,2,23)$. It is possible to expand eq.(23) for small deformations $\xi / R_{0}<<1$, in its Taylor series in all its three arguments $\xi, \xi_{\theta}, \xi_{\phi}$, around 0. By introducing the shape function,eq.(2) in eq.(23) we obtain, in the third order

$$
\begin{aligned}
& U_{S}[\eta]=\sigma R_{0}^{2} \int_{0}^{2 \pi}\left[2 S_{1,0}^{1} \eta+\left(S_{1,0}^{1}+\frac{1}{2} S_{0,1}^{1}\right) \eta^{2}\right. \\
& \left.+\frac{1}{2} S_{1,2}^{1} \eta^{3}+\frac{1}{2} S_{2,0}^{-1} \eta_{\phi}^{2}\right] d \phi+\mathcal{O}_{3}\left(\eta, \eta_{\phi}\right)
\end{aligned}
$$

where $\mathcal{O}_{3}\left(\eta, \eta_{\phi}\right)$ represents the contributions of terms involving higher orders than 3 in the function $\xi$ and its derivatives. We introduce the notations

$$
S_{n, m}^{k}=\int_{0}^{\pi} g^{n} g_{\theta}^{m} \sin ^{k} \theta d \theta
$$


The term proportional with $\eta \eta_{\phi}^{2}$ in eq.(23) is neglected because it belongs to the fourth order, since the second derivative to the square introduced a factor of $\eta_{0}^{3} / L^{2}$ which is smaller compared with $\eta_{0}^{3}$, ( $\eta_{0}$ being the amplitude of the perturbation).

The volume conservation condition

$$
V_{0}=\int_{\Sigma} \frac{r^{3}(\theta, \phi)}{3} d \Omega=\frac{4 \pi}{3} R_{0}^{3},
$$

leads, according with eqs. $(1,2)$ to the restriction

$$
\int_{\Sigma}\left(3 \xi+3 \xi^{2}+\xi^{3}\right) d \Omega=\int_{0}^{2 \pi}\left(3 A_{1,0}^{1} \eta+3 A_{2,0}^{1} \eta^{2}+A_{3,0}^{1} \eta^{3}\right) d \phi=V_{\text {cluster }} .
$$

The second term in the potential energy is given by the Coulomb interaction, for constant charge density of the nucleus $\rho_{e l}$, in the volume $V_{0}$

$$
U_{C}[\eta]=\frac{\rho_{e}}{2} \int_{\mathcal{V}}^{\prime} \int_{\mathcal{V}} \frac{1}{|\vec{r}-\vec{r}|} d \mathcal{V} d \mathcal{V}^{\prime}
$$

The Coulomb energy contains three terms: the self energy of the core of radius $R_{0}$ and of the bump (the deformation) and the interaction energy between these. Adding these terms, subtracting the Coulomb energy of the initial spherical configuration and taking into account the terms up to the third order in $\xi$, we can write the Coulomb energy in the form, $[6]$

$$
U_{C}=U_{C}^{(1)}\left[1+\left(C_{1,0}^{1}+C_{1,1}^{1}\right) \int_{0}^{2 \pi} \eta d \phi+C_{2,0}^{1} \int_{0}^{2 \pi} \eta^{2} d \phi+C_{3,1}^{1} \int_{0}^{2 \pi} \eta^{3} d \phi\right]+U_{C}^{(0)},
$$

where

$$
\begin{gathered}
U_{C}^{(1)}=3.093\left(Z_{0}-Z_{c l}\right)^{2}\left(\rho \mathcal{V}_{0}\right)^{-1 / 3}, \\
U_{C}^{(0)}=-0.665 Z_{0}^{2}\left(\rho \mathcal{V}_{0}\right)^{-1 / 3}+8.6275 Z_{c l}^{2}\left(\rho \mathcal{V}_{0}\right)^{-1 / 3},
\end{gathered}
$$

are constants and the Coulombian energy is given in MKS units. The numbers $Z_{0}, Z_{c l}$ represent the atomic mass numbers of the parent nucleus and of the corresponding cluster, respectively. The coefficients $C_{i j}$ in eq.(48) are defined in the form

$$
C_{i j}^{k}=\frac{1}{R_{0}^{i}} \int_{0}^{\pi} h^{i} g^{j} \sin ^{k} \theta d \theta .
$$


The shell energy is introduced by considering that the main contribution to the shell effects in cluster decays is due to the final nucleus, close to the double magic nucleus ${ }^{208} \mathrm{~Pb}$. The spherical core $r \leq R_{0}-h$ represents the final nucleus, which is also unexcited for the even-even case. We introduce the shell energy like a measure of the overlap between the core and the final nucleus, on one side, and between the final emitted cluster and the bump, on the other hand

$$
E_{\text {sh }}=\frac{V_{\text {over }}}{V+\left[V_{0}-\left(V_{\text {cluster }}+V_{\text {layer }}\right)\right]-V_{\text {over }}},
$$

where $V_{\text {over }}$ denotes the volume of the overlap between the volumes of the initial $V_{0}$ and final $V$ nuclei, $V_{\text {cluster }}$ is the soliton volume, eq.(46), and $V_{\text {layer }}$ is the layer volume on which the soliton is moving (i.e. $r \in\left[R_{0}-h, R_{0}\right]$ ). We use this form for the shell energy multiplied with a constant $U_{0}$, choosen such that the total energy of the system in the state of rezidual nucleus + cluster to be degenerated with the ground state energy.

The kinetic energy is given by

$$
T=\frac{\rho}{2} \int_{\mathcal{V}} v^{2} d \mathcal{V}=\frac{\rho}{2} \int_{\mathcal{V}}|\nabla \Phi|^{2} d \mathcal{V}=\frac{\rho}{2} \oint_{\Sigma} \Phi \nabla \Phi \cdot d \vec{S},
$$

where $d \vec{S}=R_{0}^{2}\left(1+\xi_{\theta}^{2}+\xi_{\phi}^{2}\right)^{-1 / 2}\left(1,-\xi_{\theta},-\xi_{\phi}\right) \sin \theta d \theta d \phi$ is the oriented surface element of $\Sigma$, eq.(1). Explicitly

$$
T=\frac{\rho}{2} \int_{\Sigma} \frac{\Phi \Phi_{r}-\frac{\Phi \Phi_{\theta} \xi_{\theta}}{r}-\frac{\Phi \Phi_{\phi} \xi_{\phi}}{r \sin \theta}}{\sqrt{1+\xi_{\theta}^{2}+\xi_{\phi}^{2}}} d S
$$

where $d S=R^{2} \sin \theta d \theta d \phi$ is the scalar surface element. Now we use the first boundary condition. By taking $\Phi_{r}$ from eq.(16) and introducing it in eq.(53) the dependence of the kinetic energy of the tangential velocity along $\theta$-direction $\Phi_{\theta}$ becomes negligible and we obtain

$$
T=\frac{R_{0}^{2} \rho}{2} \int_{0}^{\pi} \int_{0}^{2 \pi} \frac{R_{0} \Phi \eta_{t} \sin \theta+\frac{1}{R_{0}} \xi_{\phi} \Phi \Phi_{\phi}(1-\sin \theta)}{\sqrt{1+\xi_{\theta}^{2}+\xi_{\phi}^{2}}} d \theta d \phi,
$$

where we have approximated $\left.r\right|_{\Sigma} \simeq R_{0}$ in the numerator, since the corresponding higher corrections will occure in higher order than three in $\xi$. We use now the second boundary condition, which, together with the first, gives the form of the functions $f_{n}$ in the 
structure of $\left.\Phi\right|_{\Sigma}$. In the following we take the expression of $\Phi$ from eq.(9), with its coefficients $f_{n}$ obtained in eqs. $(12,14,18,21)$ and using the derivatives of $\Phi$ from eqs.(16), we have for the kinetic energy in eq.(54), in the second order in $\xi$

$$
\begin{gathered}
T[\eta]=\frac{R_{0}^{6} \rho V}{2}\left(C_{-1,2}^{3} \int_{0}^{2 \pi} \eta_{t} \int_{0}^{\phi} \eta^{2}(\tilde{\phi}) d \tilde{\phi} d \phi+2 C_{-1,2}^{3} \int_{0}^{2 \pi} \eta_{t} \int_{0}^{\phi} \eta(\tilde{\phi}) d \tilde{\phi} d \phi\right) \\
+\frac{R_{0}^{7} \rho V^{2}}{2}\left(\left(C_{-2,3}^{5}-C_{-2,3}^{6}\right) \int_{0}^{\pi} \eta \eta_{\phi} \int_{0}^{\phi} \eta(\tilde{\phi}) d \tilde{\phi}\right) d \phi
\end{gathered}
$$

where $C_{i j}^{k}$ are defined in eq.(50) and the symbol $\tilde{\phi}$ is a dummy variable of integration. Since we are looking only for traveling wave solutions (which include any sort of oscillations or moving bumps) we can transform the derivative with respect to time into the derivative with respect to $\phi$, by using eq.(2), i.e. $\partial_{t}=-V \partial_{\phi}$. We perform then an integration by parts for those term containing the primitives of $\eta$, and we finaly obtain for the kinetic energy

$$
T[\eta]=T^{(1)} \int_{0}^{2 \pi} \eta^{2} d \phi+T^{(2)} \int_{0}^{2 \pi} \eta^{3} d \phi
$$

with the parametric functions $T^{(i)}$ given by

$$
\begin{gathered}
T^{(1)}=\frac{R_{0}^{6} \rho V^{2}}{2} C_{-1,2}^{3}, \\
T^{(2)}=\frac{R_{0}^{6} \rho V^{2}}{2}\left(2 C_{-1,2}^{3} R_{0}+C_{-2,3}^{5}+R_{0} C_{-2,3}^{6}\right) .
\end{gathered}
$$

The total energy of the system is the sum of the terms given in eqs. $(43,48,51,56)$. We write it, in the second order, as a functional depending on the unknown functions $\eta(\phi, t), g(\theta), h(\theta)$ and the free parameter $V$

$$
\begin{gathered}
E[\eta, g(\theta), h(\theta)]=\int_{0}^{2 \pi}\left[\left(2 \sigma R_{0}^{2} S_{1,0}^{1}+U_{C}^{(1)}\left(C_{1,0}^{1}+C_{1,1}^{1}\right)\right) \eta\right. \\
+\left(\sigma R_{0}^{2}\left(S_{1,0}^{1}+\frac{1}{2} S_{0,1}^{1}\right)+U_{C}^{(1)} C_{2,0}^{1}+T^{(1)}\right) \eta^{2}+\left(+\frac{\sigma R_{0}^{2}}{2} S_{1,2}^{1}+U_{C}^{(1)} C_{3,1}^{1}+T^{(2)}\right) \eta^{3} \\
\left.+\frac{\sigma R_{0}^{2}}{2} S_{2,0}^{-1} \eta_{\phi}^{2}\right] d \phi+E_{\text {sh }}\left[V_{\text {cluster }}\right]+\text { const. }
\end{gathered}
$$


We note that in the deduction of eq.(58) we have implicitely used the boundary conditions, in the expression of $T$. This functional may be interpreted as depending on $\eta$ only, the function $g(\theta)$ being fixed through the conditions 1-3. Generally, the expression $E$ in eq.(58) depends on three functions $\eta(\phi-V t), g(\theta)$ and $h(\eta)$, in the third order of approximation. In order to formulate eq.(58) as the conservation law of the energy, i.e. the functional $E$ to describe a Hamiltonian functional, we use the abstract definition of a Hamiltonian system as the Lie algebra of the Poisson bracket

$$
\left\{F_{1}, F_{2}\right\}=\int_{0}^{\pi} \int_{0}^{2 \pi}\left(\frac{\delta F_{1}}{\delta \xi} \frac{\partial}{\partial \phi} \frac{\delta F_{2}}{\delta \xi}+\frac{\delta F_{1}}{\delta \xi} \frac{\partial}{\partial \theta} \frac{\delta F_{2}}{\delta \xi}\right) \sin \theta d \theta d \phi,
$$

where $F_{i}=\iint f_{i}\left(\xi^{k}(\theta, \phi, t)\right) \sin \theta d \theta d \phi$ are generic functionals and $\xi^{k}$ represents the set of the function $\xi$ together with its derivatives with respect to $\theta, \phi$ up to a certain order [12]. If we take the energy expression, eq.(58) as a Hamiltonian, $E \rightarrow H[\eta]$, then the time derivative of any quantity $F[\eta]$ is given by

$$
F_{t}=[F, H]
$$

We analyse eq.(60) for $\xi(\theta, \phi, t)=g(\theta) \eta(\phi-V t)$, i.e. the case investigated in section 2 , eq.(2), with respect to the variation of the function $\eta$ only, and for fixed $g$ and $h$ introduced as parametric functions. We define $F=\int_{0}^{2 \pi} \eta(\phi-V t) d \phi$ and, since $\delta F / \delta \eta=1$, we get from eqs. $(58,60)$

$$
\frac{d F}{d t}=\int_{0}^{2 \pi} \eta_{t} d \phi=\int_{0}^{2 \pi} \partial_{\phi} \frac{\delta\left(\mathcal{A} \eta+\mathcal{B} \eta^{2}+\mathcal{C} \eta^{3}+\mathcal{D} \eta_{\phi}^{2}\right)}{\delta \eta} d \phi
$$

where $\mathcal{A}=2 \sigma R_{0}^{2} S_{1,0}^{1}+U_{C}^{1}\left(C_{1,0}^{1}+C_{1,1}^{1}\right), \mathcal{B}=\sigma R_{0}^{2}\left(S_{1,0}^{1}+\frac{1}{2} S_{0,1}^{1}\right)+U_{C}^{1} C_{2,0}^{1}+T^{(1)}, \mathcal{C}=$ $\frac{\sigma R_{0}^{2}}{2} S_{1,2}^{1}+U_{C}^{(1)} C_{3,1}^{1}+T^{(2)}$ and $\mathcal{D}=\frac{\sigma R_{0}^{2}}{2} S_{2,0}^{-1}$, from eqs.(58). Eq.(61) gives

$$
\int_{0}^{2 \pi} \eta_{t} d \phi=\int_{0}^{2 \pi}\left(2 \mathcal{B} \eta_{\phi}+6 \mathcal{C} \eta \eta_{\phi}-2 \mathcal{D} \eta_{\phi \phi \phi}\right) d \phi
$$

Eq.(62) lead to the KdV equation, similar with the Euler approach in section 2. Therefore we have shown that the energy of the nonlinear liquid drop model, eq.(58), can be interpreted as the Hamiltonian of a of the one-dimensional KdV equation [5,7-10,13,14], in agreement with the result obtained in section 2, eq.(30), from the Euler equation approach. The coefficients of the terms in the above expression depend on two arbitrary 
functions but of different argument, and hence they represent a parametric dependence and are not involved in the Hamiltonian dynamics of the function $\eta(\phi, t)$ describing the traveling wave profile in the direction of propagation. Similar de-coupling of the coordinates is used in the theory of 2-dimensional solitary wave as in the Kortweg-Petviashvili (KP) equation $[12,13]$.

\section{The cnoidal and solitary wave solutions}

The KdV equation in its most general form

$$
A \eta_{t}+B \eta_{\phi}+C \eta \eta_{\phi}+D \eta_{\phi \phi \phi}=0
$$

has three classes of exact solutions, depending on the three initial conditions, for the same values of its coefficients, $A, B, C, D$. The most general steady-state solution of the $\mathrm{KdV}$ eqution has the form of an oscillation that can reduce to the simple-pulse solution in the limit that the oscillations period tends to infinity. This solutions can be classified using the phase space portrait, Fig.1, in cnoidal waves, solitary waves and singular solutions, Fig.2. In the following we denote with $V$ the traveling velocity (phase velocity), with $L$ the half-width and with $\eta_{0}$ the amplitude of the solutions, eqs. $(32,33)$. For small amplitudes $\left|\eta_{0}\right| \ll 1$, i.e. $C \simeq 0$, the $\mathrm{KdV}$ equation with has approximate solutions in the form of stationary small amplitude oscillations, if $\operatorname{sign}(C D)=+1$. This is the case when the nonlinear term (the coefficient $C$ in eq.(31)) is neglected, the dispersion law becomes $L=2 \pi / V^{3 / 2}$ and the group velocity is $V_{g r}=3 V$. These solutions are instable and fall into cnoidal waves, if their amplitude is increased with the increasing of the energy.

The algebraic convenience is obtained by writing the steady state solutions in the form of eq.(2) with $\eta_{t}=-V \eta_{\phi}$. This allows us to use, in the following, the variable $z=\phi-V t$. The KdV equation reduces to an ordinary differential equation for which a first quadrature may be immediatley obtained. A second quadrature is possible, after 
the first one, by multiplication of the resulting equation with $\eta_{z}$. We find that

$$
\eta_{z}^{2}=\frac{2 C}{3 D} \eta^{3}+\frac{A V-B}{D} \eta^{2}+a \eta+b,
$$

where $a, b$ are constants of integration. Eq.(64) factorizes into

$$
\eta_{z}^{2}=-4\left(\eta-\alpha_{1}\right)\left(\eta-\alpha_{2}\right)\left(\eta-\alpha_{3}\right)=-U[\eta]
$$

with $\alpha_{1} \leq \alpha_{2} \leq \alpha_{3}$ real roots and with the same interpretation for $U[\eta]$ like in eq.(58) and Figs.1, of the kinetic (LHS of eq.(62)), potential $(U)$ and total $(E)$ energies. Since $\eta_{z}$ is real, for finite amplitude oscillations $\eta$ must be confined to the range $\alpha_{2} \leq \eta \leq \alpha_{3}$, Fig.3. In between that range, the potential energy has a valley which characterizes the stability of the solutions. Setting

$$
\alpha_{3}-\eta\left(\sqrt{\frac{\left(\alpha_{3}-\alpha_{1}\right) C}{6 D} z}\right)=\left(\alpha_{3}-\alpha_{2}\right) w^{2}(z)
$$

we obtain the differential equation

$$
w_{z}^{2}=\left(1-w^{2}\right)\left(1-k^{2} w^{2}\right)
$$

with $m^{2}=\frac{\alpha_{3}-\alpha_{2}}{\alpha_{3}-\alpha_{1}}$. This equation defines the Jacobi elliptic functions $s n(z \mid m)$ of parameter $m$ of amplitude $z$ [15]

$$
\operatorname{sn}(z \mid m)=\sin \beta, \quad z=\int_{0}^{\beta} \frac{d x}{\sqrt{1-m \sin ^{2} x}},
$$

and evidently $z(\pi / 2, m)=K(m), c n^{2}(z \mid m)=1-\operatorname{sn}(z \mid m)$. The square of the cnoidal sinus and cosinus oscillates between 0 and 1 , when $m$ takes real values in $[0,1]$, with a period equal with $2 K(m)$, where

$$
K(m)=\int_{0}^{1} \frac{d x}{\left[\left(1-x^{2}\right)\left(1-m^{2} x^{2}\right)\right]^{1 / 2}},
$$

represents the Jacobi elliptic integral. The solutions become

$$
\eta(\phi-V t)=\alpha_{2}+\left(\alpha_{3}-\alpha_{2}\right) c n^{2}\left(\sqrt{\frac{C\left(\alpha_{3}-\alpha_{1}\right)}{6 D}}(\phi-V t) \mid m\right) .
$$

The solution $\eta$ oscillates between $\alpha_{2}$ and $\alpha_{3}$, with a period $T=2 K(m) \sqrt{\frac{6 D}{\left(\alpha_{3}-\alpha_{1}\right) C}}$, as one can see from the negative potential valley in Fig.3. This solution is not allowed 
to exist outside of this valley. However, the shape of the valley can be modified by choosing different values for the parameters $\alpha_{i}$, i.e. one can associate different potential pictures to different sets of initial conditions. The function $c n(z \mid m)$ has two limiting forms $c n(z \mid 0)=\cos z, c n(z \mid 1)=\operatorname{sech} z$, see Fig.2, too. The potential for both these limits is presented in Fig.3. If $\alpha_{2}$ approaches $\alpha_{1}, m$ approaches 1 and $T, K \rightarrow \infty$. We obtain in this limit a localised solution

$$
\eta(\phi-V t)=\alpha_{2}+\left(\alpha_{3}-\alpha_{2}\right) c n^{2}\left(\sqrt{\frac{C\left(\alpha_{3}-\alpha_{2}\right)}{6 D}}(\phi-V t) \mid 1\right),
$$

which is a bump of half-width $L=\sqrt{\frac{6 D}{C\left(\alpha_{3}-\alpha_{2}\right)}}$ and amplitude $\alpha_{3}-\alpha_{2}$ above a reference level $\alpha_{2}$. More, since $\alpha_{1}+\alpha_{2}+\alpha_{3}=\frac{3(B-A V)}{2 C}$, we can write, in the limit $\alpha_{2}=0$ (so that $\left.\alpha_{3}=\frac{3(B-A V)}{2 C}\right)$

$$
\eta(\phi-V t)=\eta_{0} \operatorname{sech}^{2}\left[\sqrt{\frac{\eta_{0} C}{12 D}}(\phi-V t)\right]
$$

which is exactly the soliton solution, i.e. eq.(32). From Fig.3 we see that the solution is defined between the limits $\alpha_{1}, \alpha_{3}$ (the maximal domain of definition) and, due to the value zero of the derivative of $\eta$ in $\alpha_{1}$ (the saddle point), it requests infinite time to approach this limit, therefore it has a high stability. On the other hand, a steady-state solution in the form of a small oscillation is obtained when $\alpha_{3} \rightarrow \alpha_{2}$ and $m \rightarrow 0, T \rightarrow$ $\pi / 2$. In this limit we have the solution for small harmonic oscillations in the form

$$
\eta(\phi-V t)=\alpha_{2}+\left(\alpha_{3}-\alpha_{2}\right) \cos ^{2}\left(\sqrt{\frac{\left(\alpha_{3}-\alpha_{1}\right) C}{6 D}}(\phi-V t)\right) .
$$

In this latter case the range for $\eta$ becomes very small, in principle a point situated in the bottom of the potential valley centered on $\alpha_{3}$, Fig.3, (but in fact a small domain, since the $\mathrm{KdV}$ equation was deduced in the second order only) In conclusion, within the same equation, only by modifying the initial conditions and the parameter $V$, one can obtain both periodic solutions and localised bumps. A picture showing the smooth deformation from the cosine function towards the soliton is presented in Fig.4. Such different choices introduce different potentials, as in Fig.3. In this way, following a certain path in the space of these parameters, the dynamical evolution can smoothly approach both limits. The solutions depend on three free parameters (constants of 
integration) subjected to two additional conditions. On one side we have the condition of the volume conservations, requested by the physical solutions, eqs. $(45,46)$. On the other side the solutions must be periodic, i.e.

$$
K \sqrt{\frac{\alpha_{3}-\alpha_{2}}{\alpha_{3}-\alpha_{1}}}=\frac{\pi}{n} \sqrt{\alpha_{3}-\alpha_{1}},
$$

for $n=1,2, \ldots, N$ with $N$ always finite $(K(m)$ is bounded from below) such that

$N \leq 2 \sqrt{\alpha_{3}-\alpha_{1}}$. Consequently the whole model contains only a free parameter which can be choosen to be one of the $\alpha$ 's, $V$ or $\eta_{0}$, and we can draw the potential shape $U\left(r, \eta_{0}\right)$ as a 2-dimensional surface. In Fig.5 we present such a contour levels potential picture where the coordinates are the amplitude of the soliton $\eta_{0}$ (denoted $r$ ) and the parameter $\alpha_{2}$, (denoted $a$ ). Each cross section of the potential surface parallel to the $r$-axis represent the potential of the solution, for a certain initial condition (a fixed value of $\alpha_{2}$ ) and a certain fixed volume. In the limit of the soliton wave ( $r$ around 3) we have a deep potential hole $\left(\alpha_{2}=\alpha_{3} \simeq 1.6\right)$ and in the limit of the small oscillations ( $r$ around 2.4, $\left.\alpha_{2}=\alpha_{1} \simeq 3.2\right)$ we obtain again a minimum, embedded into a large potential valley. The reference level of the soliton $(r \simeq 1.4)$ is smaller than the average reference level of the small oscillations, due to the volume conservation. The evolution of the corresponding solution $\eta\left(\phi-V t ; \alpha_{2}\right)$ between these limiting forms is plotted in Fig.6.

\section{$5 \quad$ The spectroscopic factors}

The spectroscopic factor are defined as the ration between the experimental decay $\left(\lambda_{\text {exp }}\right)$ over the theoretical one-body constant $\left(\lambda_{\text {Gamow }}\right)$. For the alpha decay, the relative ratios are well reproduced but the absolute values are underestimated at least by two orders. Possible explnations arrise from the fact that the many-body correlations are neglected in these approaches, the correct antisymmetrization of the channel wave function, the impossibility of separation in two factors of the transition element in the integral theory $[1,2]$. In the present paper we consider that we have introduced the many-body effects 
in a phenomenologic way, based on a collective model, resulting in the possibility of describing of the large enhancement of the alpha cluster on the nucler surface. hence, we try to give an explanation of the spectroscopic factors based on this model. By considering the cnoidal waves as solutions of both the hydrodynamic equations (Euler) and the liquid drop model equations, we obtain new shapes which show that, starting from the harmonic normal modes on the nucler surface and due to the nonlinearities, some nucleons are grouping together to form the emitted cluster. In the cnoidal wave description, the spectroscopic factors may be given by the ratios of the two wave amplitudes in the corresponding wells, evaluated with the help of barrier penetrability between the two minima, Fig.5, [4,5]. The spectroscopic factors are given by the penetrabilities of the above barriers

$$
S=\exp \left(-\frac{2}{\hbar} \int_{0}^{\eta_{0}}\left(A_{\text {cluster }}-E[\eta]\right)^{1 / 2} d \eta\right)
$$

where $\eta_{0}$ is the final amplitude of the soliton, Fig.6, . The function $E[\eta]$ is the total energy from eq.(57), with the parameter $U_{0}$ fitted such that the second minimum of the potential energy, Fig.7, to be degenerated in energy with the first one. The numerical parameters are identical with that one used in the references [4,5]. The result is compared with similar calculations $[4,5,16,17]$ and with the experimental preformation factors for ${ }^{208} \mathrm{~Pb},[\mathrm{~s}]: S_{\exp }=0.085, S_{r e f .[4,5]}=0.095, S_{B W}=0.0063$ and $S_{\text {soliton }}=0.07$. The first theoretical result was obtained in the frame of a previous form of the present model [4]. The second result represents the spectroscopic factor $S_{B W}=\left(6.3 \times 10^{-3}\right)^{\frac{A_{\text {cluster }}-1}{3}}$, obtained in [mm] by using a semiempirical heavy ion potential. However we mention that there exists some ambiguities in the definition of the spectroscopic factors.

\section{Conclusions}

In this paper we present a nonlinear hydrodynamic model describing the new large amplitude collective motion in nuclei, suggested by the new exotic alpha (and cluster) 
decays. From the Euler equation, subjected to nonlinear boundary conditions at the free surface and the inner surface of the fluid layer, we obtain, up to the third order in the deviation of the surface from the spherical shape, a dynamical equation for the radial coordinate in the form of the Korteweg de Vries eqution. The new introduced nonlinear terms lead to a model describing steady-states as knoidal waves on the surface of the nucleus. These solutions are described by the Jacobi elliptic functions and cover continuously all the range between small harmonic oscillations, anharmonic oscillations up to solitary wave. This model approaches the traditional liquid drop model in the linear approximation, when the $\mathrm{KdV}$ equation transforms into the Helmholtz eqution and the cnoidal waves approach their periodic limit, i.e. the normal modes of vibration of nuclei. From the total energy of the liquid drop model (surface, Coulombian, kinetic and shell energy), calculated in the same order of approximation, it results a functional depending on the shape functions which is exactly the Hamiltonian of the Korteweg de Vries equation, hence a Hamiltonian formalism applied to the present model lead to the same dynamical equation. The final results for the dynamical equations and the energy are in agreement with the previous (one-dimensional) version of the same model, $[4,5]$. The solitary wave limit of the solutions could describe the preformation of the clusters on the nuclear surface. We investigate the potential energy, depending on three parameters: the amplitude of the deformation, the phase velocity of the deformation, and the depth of the layer, under the restrictions of constant volume of the cluster and periodicity of the solutions on the surface. From the expression of the potential energy we find a minimum associated with the harmonic vibration, (small oscillation limit) and an additional minimum corresponding to the solitonic shapes as clusters on the nuclear surface. By choosing the shell effect contribution such that the two minima are degenerated, we obtain a potential energy profile which could describe the potential barrier associated with the preformation of such clusters. The spectroscopic factors, calculated as the ratio of the square amplitudes in the two minima, are in good agreement with the experiments. 


\section{References}

[1] A. Sandulescu, W. Greiner,Rep. Prog. Phys., 551423 (1989)

[2] P. B. Price, Ann. Rev. Nucl. Part. Sci., 3919 (1989)

[3] W. Greiner and J. Eisenberg, Nuclear Models (North-Holland, 1987)

[4] A. Ludu, A. Sandulescu and W. Greiner, Int. J. Modern Phys. E 1, 169 (1992)

[5] A. Sandulescu, A. Ludu and W. Greiner, Proc. Inter. Conf. Nucleus and Clusters, Turku, Finland, June 1991, eds. M. Brenner and F. B. Malik (Springer-Verlag, Berlin 1991), p.262; A. Ludu, A. Sandulescu and W. Greiner, Int. J. Modern Phys. E 2, 4, (1993) 855; J. Phys. G: Nucl. Part. Phys. 211715 (1995); A. Ludu,

A. Sandulescu, W. Greiner, K. M. Källmann, M. Brenner, T. Lönnroth and P. Manngärd, J. Phys. G: Nucl. Part., 21 L41 (1995); V. G. Kartavenko, K. A. Gridnev and W. Greiner, Int. J. Mod. Phys., 3, 1219 (1994); V. G. Kartavenko, A. Ludu, A. Sandulescu and W. Greiner, Int. J. Mod. Phys. E 5329 (1996)

[6] D. J. Korteweg, G. de Vries, Phil. Mag., 39 (1895) 422

[7] G.L. Lamb, Elements of Soliton Theory, (John Wiley \& Sons, New York, 1980); C. Rebbi and G. Soliani, Solitons and Particles, (World Scientific, Singapore, 1984); S. Novikov, S. V. Manakov, Theory of Solitons. The Inverse Scattering Method, (Consultants Bureau, New York, 1984); R. K. Bullough and P. J. Caudrey, Eds., Solitons, (Topics in Current Physics, Springer-Verlag, Berlin, 1980)

[8] G. Eilenberger, Solitons. Mathematical Methods for Physicists, (Springer-Verlag, Berlin, 1981)

[9] R. K. Bullogh and P. J. Caudrey, Solitons, (Springer-Verlag, Berlin, 1980); P. G. Drazin, R. S. Johnson, Solitons : an Introduction, (Cambridge University Press, Cambridge, 1989).

[10] C. Rebbi and G. Soliani, Solitons and Particles (World Scientific, Singapore, 1984) 
[11] K. Kreutz, Phys. Rev. D 123126 (1975); R. Rajaraman, Solitons and Instantons. An Introduction to Solitons and Instantons in Quantum Field Theory, (North Holland, Amsterdam, 1984)

[12] R. Abraham and J. E. Marsden, Foundations of Mechanics, (The Benjamin/Cummings Publishing Company, Inc., Reading, Massachustes, 1978); V. I. Arnold, Mathematical Methods of Classical Mechanics, (Springer-Verlag, New York, 1978)

[13] F. B. Estabrook and W. D. Wahlquist, J. Math. Phys., 171293 (1976); Y. Nogami, F. M. Toyama and S. Zhao , J. Phys. A: Math. Gen., 281413 (1995); Mohammad and M. Can, J. Phys. A: Math. Gen., 283223 (1995); H. D. Wahlquist and F. B. Estabrook, Phys. Rev. Lett. 31 1386; (1973); J. Math. Phys., 161 (1975); M. D. Kruskal, R. M. Miura, C. S. Gardner and N. J. Zabusky, J. Math. Phys., 11952 (1970); R. M. Miura, SIAM Rev. 18412 (1976) and R. M. Miura, C. S. Gardner and M. D. Kruskal, J. Math. Phys., 91204 (1968)

[14] L. D. Landau and E. M. Lifschitz, Fluid Mechanics (Pergamon Press, Oxford, 1987), § 62

[15] M. Abramowitz and I. A. Stegun, Handbook of Mathematical Functions, (Dover Publications Inc., New York, 1968)

[16] H. J. Mang, Phys. Rev. 1191069 (1960)

[17] R. Blendowske and A. Walliser, Phys. Rev. Lett 611930 (1988). 


\section{Figure Captions}

Figs.1

The potential picture and the phase space trajectories for some traveling wave solutions. We present, in the left side of Fig.1a the effective cubic potential associated with the $\mathrm{KdV}$ equation, eq.(30), and in the right side the corresponding phase space portrait with the curves describing motions of constant energy. One associates with the finite potential valley a class of periodic solutions consisting in anharmonic oscillations (a), centerd around the bottom (A) of the valley. In their superior limit these solutions are bounded by a special singular solution (the separatrix - S) of infinite period, hence a localised bump for the function $\eta(\phi) \simeq \eta(t)$. Starting from the right side of the potential valley to the saddle point (the origin of the phase space), the corrsponding motion represents the soliton solution. The right limit of the $(\mathrm{S})$ curve gives the soliton amplitude. The amplitude of the anharmonic oscillations (cnoidal wave, i.e. periodic solutions of the KdV equation) are limited by the soliton height. Eq.(30) has also other classes of singular solutions, (b-c), which have no physical semnification for the present model, i.e. not being traveling waves.

In Fig.1b we present the same picture, for the linear approximation of eq.(30). The effective potential is quadratic and gives periodic solutions, harmonic oscillations. For comparison, we ploted in the same frame the original cubic potential. One can see how the nonlinearity developes and creates the pocket and the saddle.

Fig.2

We present the three classes of solutions $(\eta(\phi-V t))$ of the $\mathrm{KdV}$ equation: the soliton solution (one soliton bump of height 12 in the figure), cnoidal waves (large and small amplitude anharmonic oscillations ), and the singular (unphysical) solutions (decreasing to $-\infty)$. The cnoidal waves are similar with some periodic copies of the

soliton shape, and the small amplitude ones are (in the presented case) just cosine 
functions.

Fig.3

The effective potential associated with the $\mathrm{KdV}$ equation is plotted against the coordinate $z$, eq.(65). The general steady-state solution $(c n(z \mid m))$ has three real zeros, $\left(\alpha_{i}\right)$, which controll the range of the amplitude. It has two limits: the soliton $(S e c h)$ and the harmonic oscillations (cos). The soliton realises the deeper potential valley, where from its stability.

Fig.4

The cnoidal cosine function $c n(z \mid m)$ ploted against its argument and the parameter $m$. For $m=1$ the function approaches the Sech limit and for $m=0$ the cnoidal cosine approaches the cos limit.

Fig.5

The contour plots of the potential surface $U[\eta]=U\left(r, \alpha_{2}\right)$ plotted for a fixed volume $V_{\text {cluster }}=4$. One can see the initial state characterized by harmonic oscillations, $\left(r=2.4, \alpha_{2}=3.2\right)$, and the final state of minimum energy associated with the soliton, $\left(r=3, \alpha_{2}=1.6\right)$.

Fig.6

The shape of the surface associated with the general solution of the KdV equation, plotted against the coordinate $r=R_{0}(1+\eta(\theta))$ and the parameter $V$. The smooth transition from the harmonic oscillations form $(V \simeq 0)$ to the final solitonic form $(V \simeq 2)$, under the volume conservation restriction, is presented. 


\section{Fig.7}

The potential barrier around a path of minimum energy, in the plane of parameters $\eta_{0}, \alpha_{2}$, from the small oscillations (first minimum of zero energy) to the solitonic solution (second minimum). The higher curve represents the energy without the term $E_{s h}$. The curve which has the second minimum degenerated in energy with the first one, represents the total energy, with the shell corrections included. Along the path therer are labeled the corresponding values of $\eta_{0}$. 

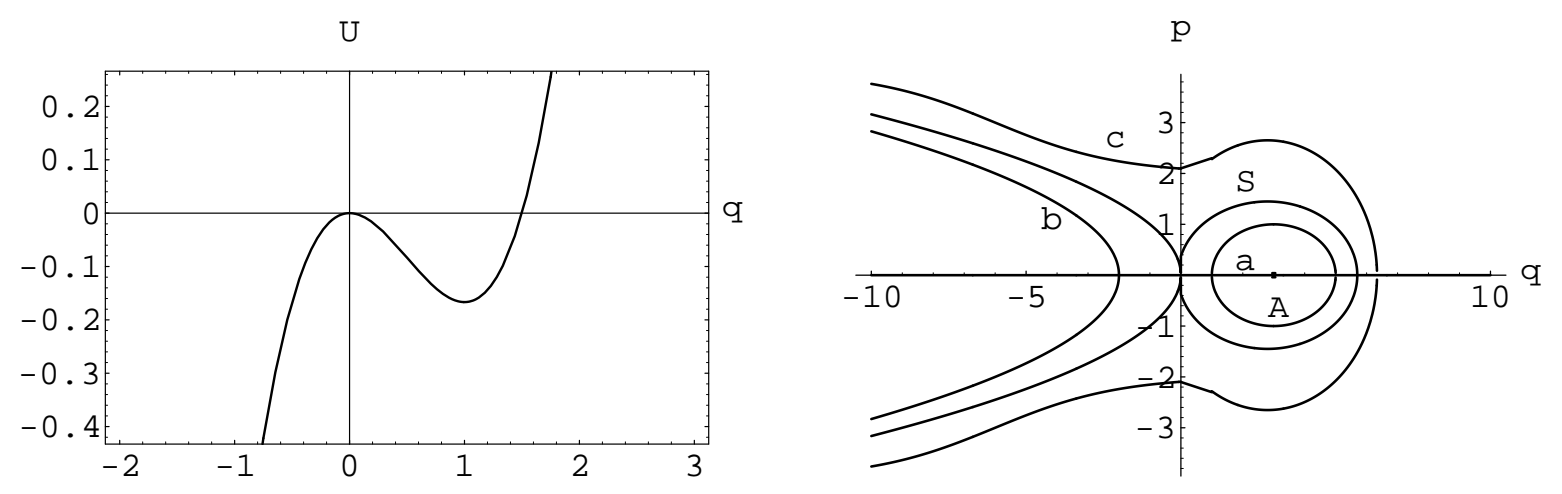

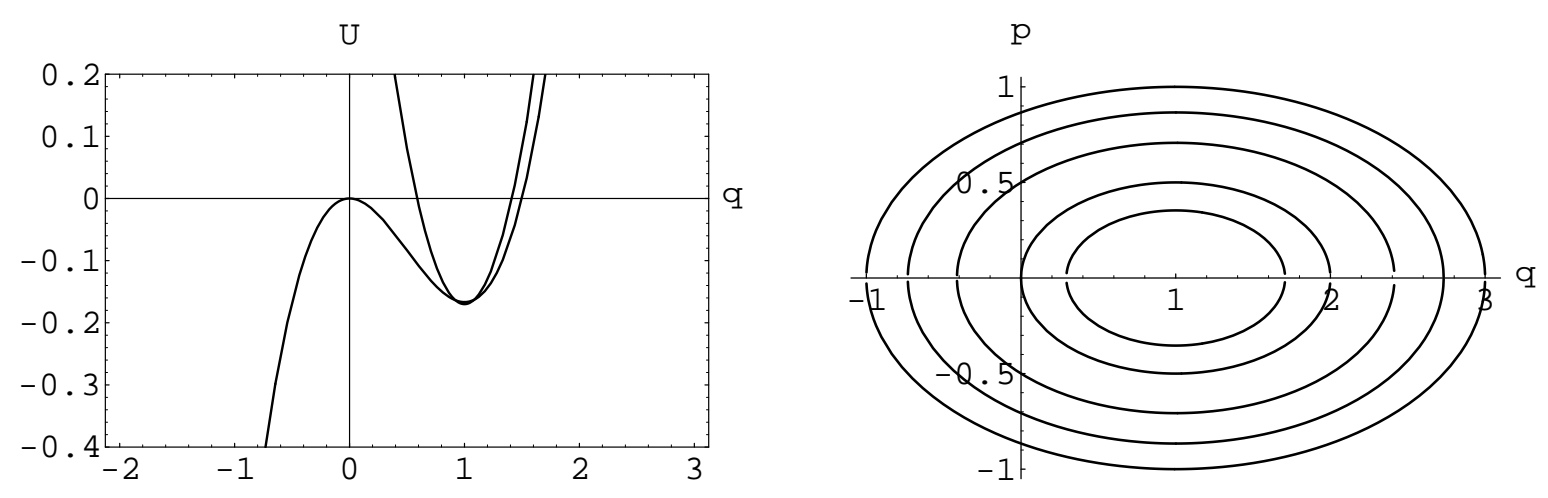


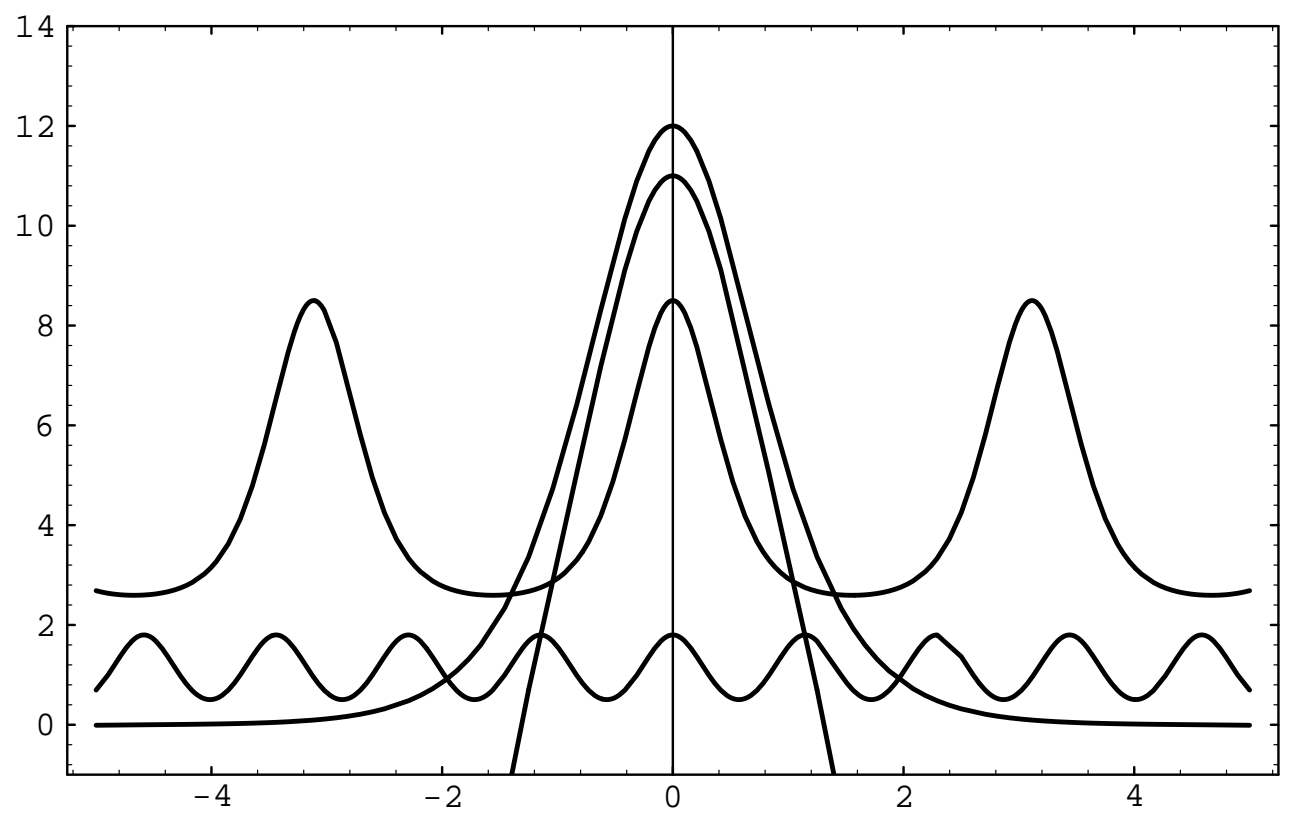




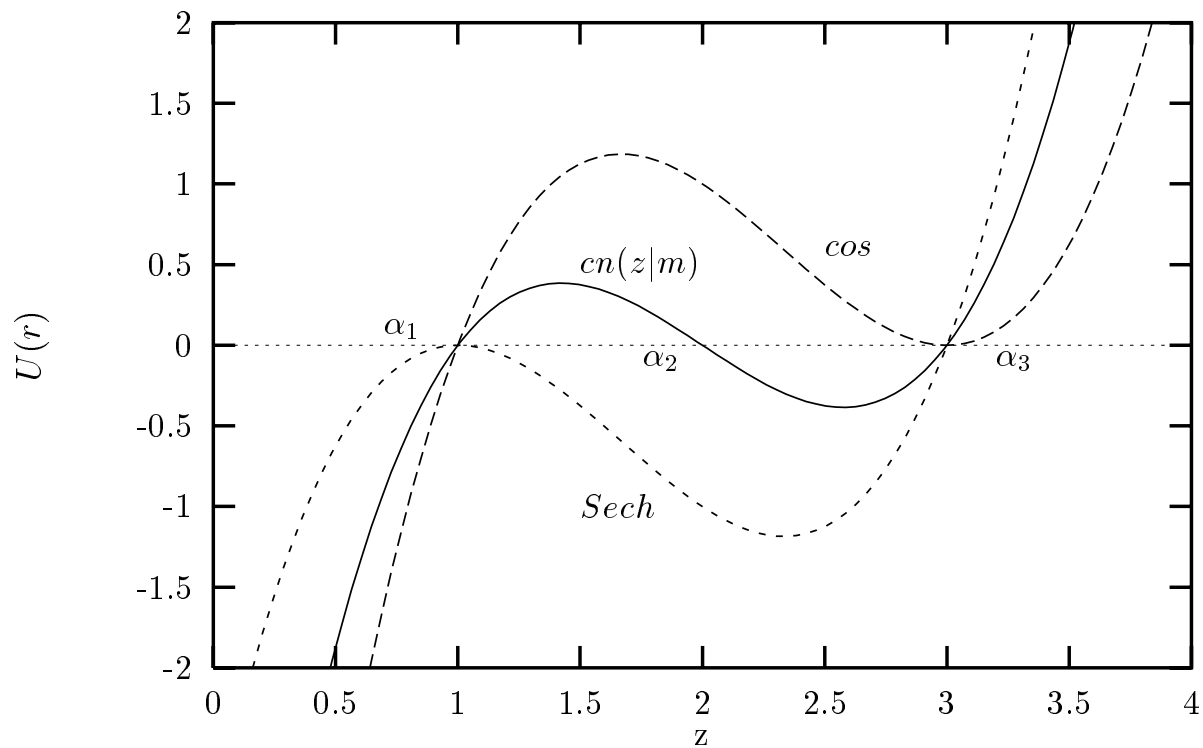




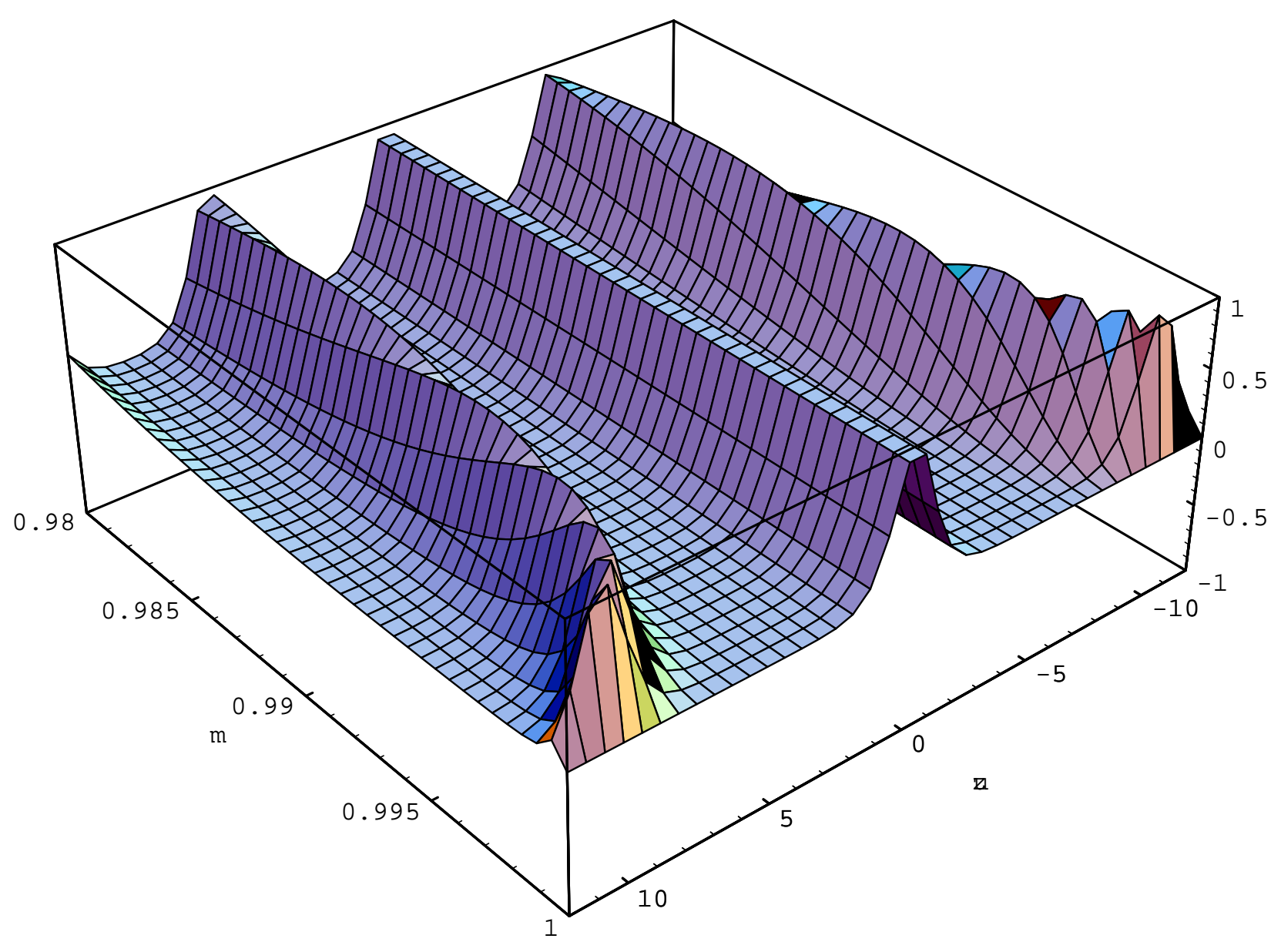




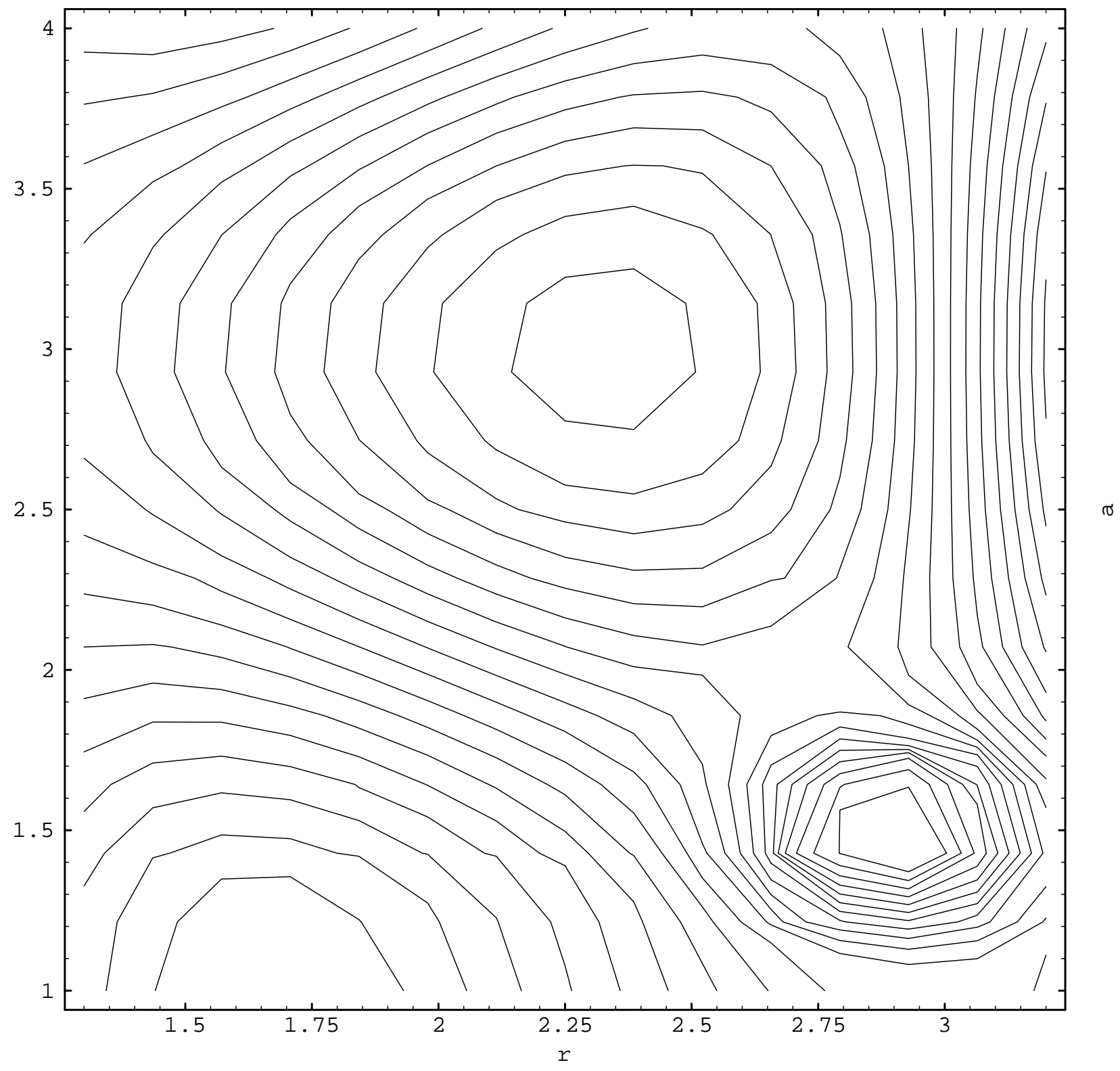




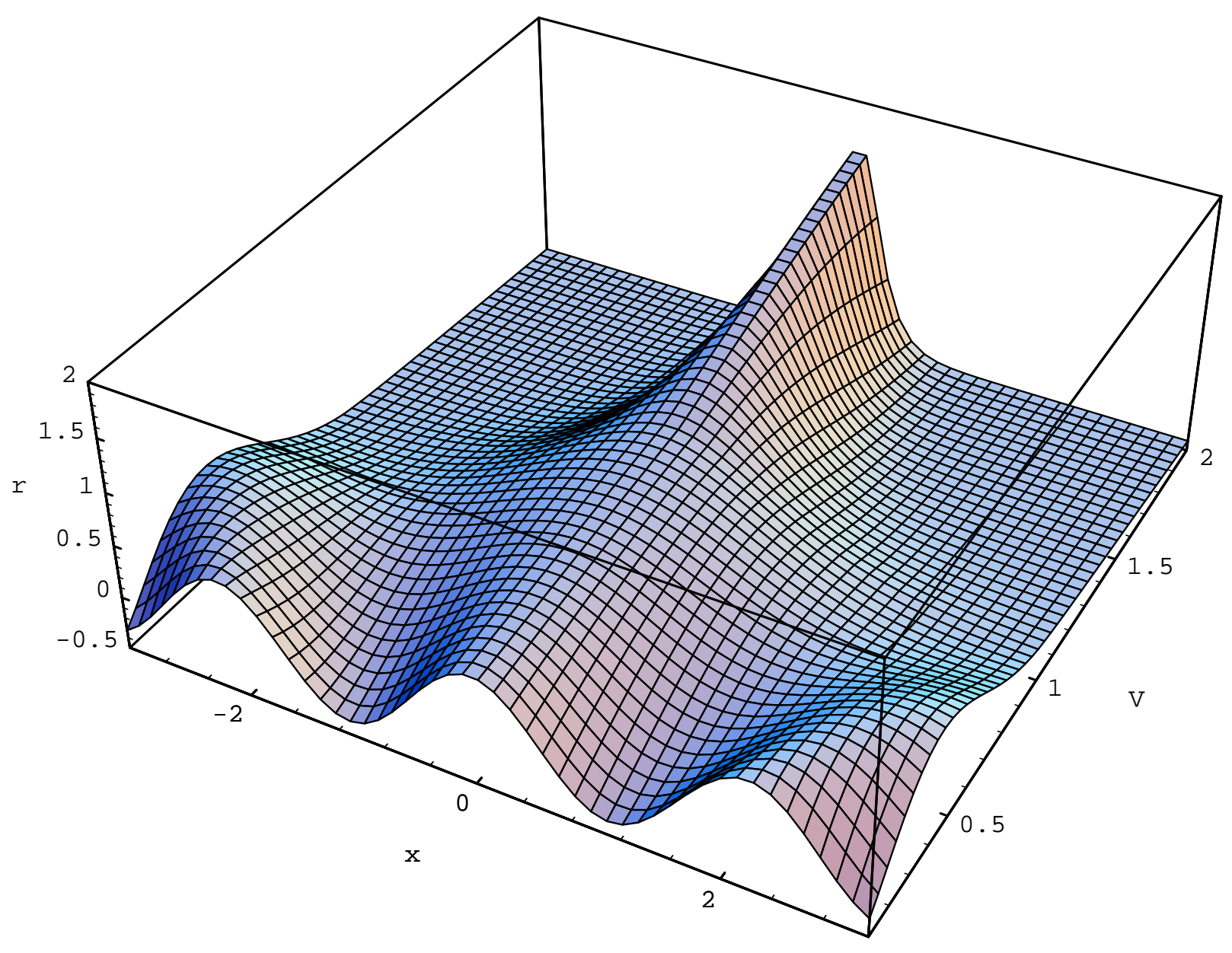


$\mathrm{U}[\mathrm{MeV}]$

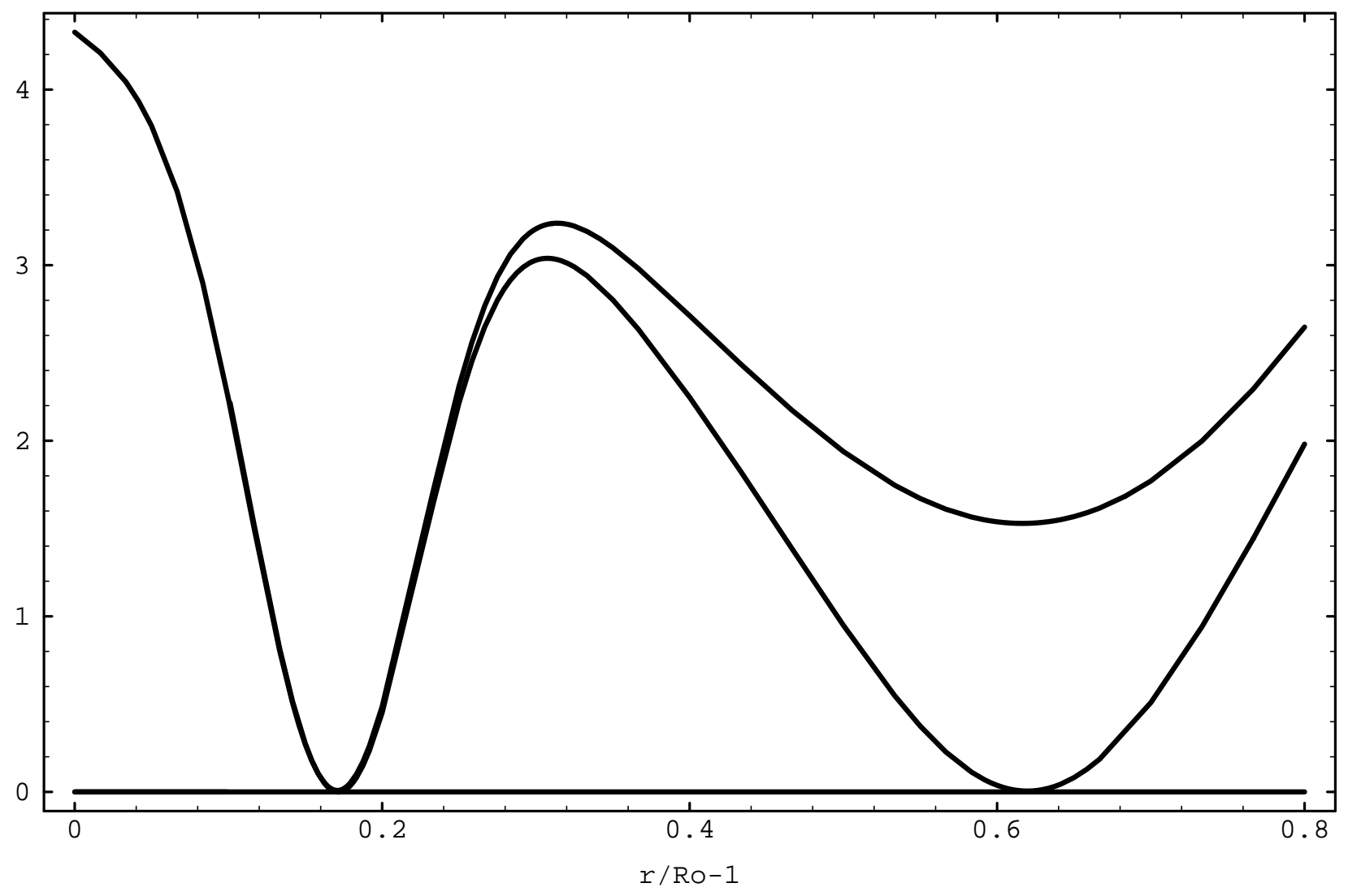

